\title{
Strategy of Harmonic Suppression of Smart DistributionNetwork with Grid-Connected Photovoltaic Generation
}

\author{
Lifeng Zhu, Shuxi Xu, Zijia Ding, Lijun Qin, and Feng Jiao
}

\begin{abstract}
In this paper, the main impact of harmonics current of PV generation on smart distribution network a fter it is connected with the latter is indicated, and the reasons for the impact are analyzed. The methods of system harmonic suppression is summarized, three methods of $P V$ power system based on active power filter, reactive power filter and combined system of static var compensator and active power filter are studied separately. Finally, according to the development of smart distribution network and application research on PV generation, the existing problems of the PV grid-connected system with harmonic suppression function are pointed out and its study direction in the future is also looked ahead.
\end{abstract}

Index Terms-APF, combined compensation, grid connected, harmonic suppression, photovoltaic generation, smart distribution network.

\section{INTRODUCTION}

Recently, photovoltaic (PV) application has received a great attention in research because it appears to be one of the most efficient and effective solutions to environmental problems. As a kind of important distributed power, PV power generation is gradually from the independent system to large sale grid-connected system in direction, and the number of grid-connected PV systems has had an exponential growth. Supporting the reasonable access of a large number of distributed powers is one of the important features of smart distribution network, which not only has less investment, flexible power generation, environmental compatibility but also improve and perfect the demand side response and management functions, completely conforming to the development requirements of the smart distribution network framework [1].

However, the PV grid-connected system usually operates in low utilization due to the characteristics of intermittency and uncertainty, PV grid-collected inverter is easy to produce the problem of harmonic and three phase current unbalance, the harmonic problem is one of the most series sad problems to restrict photovoltaic grid-collected power station to attain near sinusoidal outputs of the grid-connected photovoltaic system, to improve the power

Manuscript received October 18, 2012; revised November 20, 2012. This work was supported in part by the International Conference on Power Science and Engineering.

Lifeng Zhu is with the Shandong Electric Power Group Corporation, J ining, China. (e-mail: jngdfz@163.com).

Shuxi Xu, Zijia Ding, Lijun Qin and Feng Jiao are with North China Electric Power University, Beijing, China. (e-mail: jiao3395@ sohu.com). quality, this paper goes into depth on some problems of harmonic suppression in smart distribution network with grid-connected photovoltaic generation.

\section{MAIN IMPACT OF HARMONIC PROBLEMS}

Photovoltaic grid-collected inverter is easy to produce the problem of harmonic and three phase current unbalance, the harmonic problem is one of the most series problems to restrict photovoltaic grid-collected power station, and high harmonics exist in many PV station, especially in low light conditions[2]. The mechanism of the harmonic formation and interaction is complex, the two factors as follow:

The control technology of switch tubes opening delay is adopted in the grid-connected inverter link in practical application, produced a control dead band time. The control dead band makes the output of inverter bridge arm stack a voltage deviation. The voltage deviation is decided by the flow direction of output current, not controlled by switch tubes, causing harmonics directly[3].

Resonance phenomenon is existed in T filter itself, easy to cause the system unstable. At the same time, harmonic current produced by PWM inverter link may be incentive to filter resonances, which result in the harmonic amplitude increases suddenly.

\section{HARMONIC SUPPRESSION OF PV POWER SYSTEM BASED ON APF}

APF and photovoltaic grid generation unified control system can collect the function of harmonic and reactive current compensation and photovoltaic grid-connected system. The working principle is described briefly as follows: the current detection part is proposed to detect the reactive and harmonic compensate current of nonlinear load. Maximum power point tracking (MPPT) could deliver the great advantages of solar power to the grid, forming grid-connected instruction current[4]. The control part combines the reactive and harmonic compensate current and grid-connected instruction current to form the new instruction current, which is the basis of the current flowing from the convertor to grid through suitable control method. It can not only realize current quality management and photovoltaic grid generation. The key problem to harmonic compensation is that two points: extraction of reactive power and harmonic current compensation signal and instruction signals synthesis. 


\section{INSTRUCTION SignALS DETECTION}

The harmonic and reactive current detection method is an important research branch of APF, theory of instantaneous reactive power is currently the most widely used in the APF diction methods, which two kinds of harmonic current detecting method are put forward on this basis. The core idea of the theory of instantaneous reactive power is to transform instantaneous value of each phase voltage and current to the alpha and beta orthogonal coordinate system through transformation matrix. The dot product of the voltage and current vector is defined as instantaneous active power, the fork product of the voltage and current vector as instantaneous reactive power [5]. On this basis, widely used p-q method and ip-iq method are developed. The advantage of detection methods based on the theory of instantaneous reactive power is to realization circuit simpler, less delay, good real-time, however, exiting large amount of calculation, vector transform complex in application, only applied to three-phase system.

Different from the three phase photovoltaic grid system, the harmonic detection method based on the theory of instantaneous reactive power can't be directly applied in single phase system. There are some researches for single phase harmonic detection at home and abroad, such as making use of single-phase circuit to structure virtual three-phase circuit in accordance with the difference of each phase.

\section{AlgORITHM OF INSTRUCTION SIGNALS SYNTHESIS}

The instruction current synthesis refers to the composite operation of active power instruction current of grid-connected photovoltaic power generation and the complement instruction current of reactive power and harmonic current. According to the theory of instantaneous reactive power, the fundamental wave active dc component can be acquired after three phase load current flow through transformation matrix and low pass filter [6]. The expression of instruction current is as follow, $i_{p}^{*}$ is $\mathrm{dc}$ instruction component of grid-connected photovoltaic power generation, the fundamental wave active dc component stacks with $i_{p}^{*}$, then the current flows through transformation matrix to achieve the fundamental wave active component of load current $i_{a p f}, i_{b p f}, i_{c p f}$.

$$
\begin{aligned}
& {\left[\begin{array}{c}
i_{a}^{*} \\
i_{b}^{*} \\
i_{c}^{*}
\end{array}\right]=\left[\begin{array}{l}
i_{a} \\
i_{b} \\
i_{c}
\end{array}\right]-\left[\begin{array}{l}
i_{a p f} \\
i_{b p f} \\
i_{c p f}
\end{array}\right]=\left[\begin{array}{l}
i_{a}(t)-\sqrt{\frac{2}{3}} \sin \omega t \overline{i_{p}(t)} \\
i_{b}(t)-\sqrt{\frac{2}{3}} \sin \left(\omega t-\frac{2}{3} \pi\right) \overline{i_{p}(t)} \\
i_{c}(t)-\sqrt{\frac{2}{3}} \sin \left(\omega t+\frac{2}{3} \pi\right) \overline{i_{p}(t)}
\end{array}\right]-} \\
& \sqrt{2 / 3}\left[\begin{array}{l}
i_{p}^{*} \sin \omega t \\
i_{p}^{*} \sin \left(\omega t-\frac{2}{3} \pi\right) \\
i_{p}^{*} \sin \left(\omega t+\frac{2}{3} \pi\right)
\end{array}\right]
\end{aligned}
$$

In order to make full use of photovoltaic grid inverter capacity, to improve the system response speed and realize the load harmonic and reactive current compensation, the corresponding control methods can be taken I accordance with the varies of load and different types.

As shown in Fig. 1, instruction signals synthesis based on load identification judges the performance of load. If light, the fundamental and harmonic current needed by load is provided completely by photovoltaic grid, so that the link of harmonic detection can be ellipsis to improve the system response speed; if overloaded, the reactive power and harmonic signals would be extracted to judge whether it is beyond the allowance of the rated current of the inverter or not [7]. The signal can be directly as a command signal to synthesize with active signal when it is in the permissible range or less than. If not, the two methods can be carried on the limiting control: amplitude clamping algorithm and amplitude scaling algorithm.

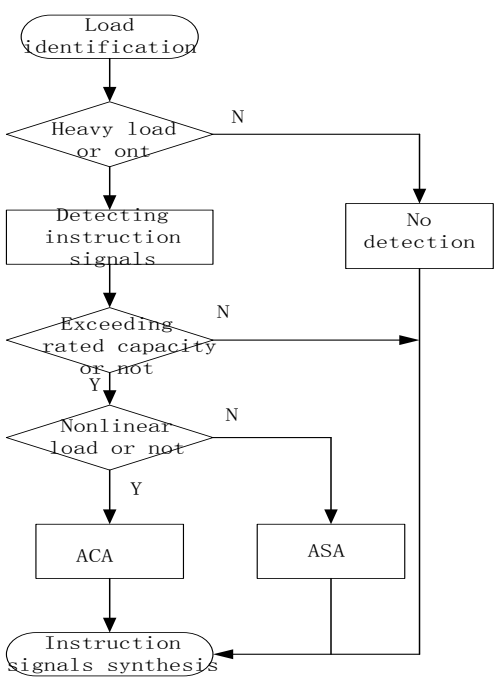

Fig. 1. Diagram of instruction signals synthesis based on load identification

\section{PASSIVE FILTER}

Passive filters are still very applicable from the view of the production cost and the effectiveness and reliability of control method, in view of working characteristics of the photovoltaic power generation system inverter [8]. A new type of triangle series-parallel passive filter device is designed as shown in Fig. 2.

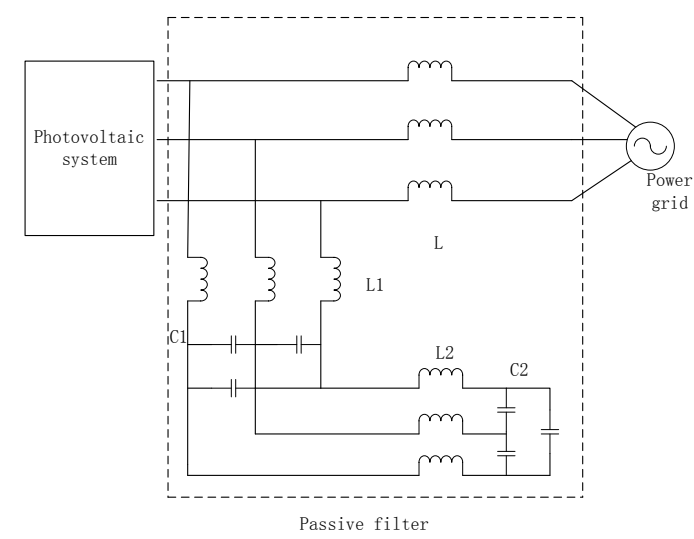

Fig. 2. Structure of triangle series parallel band-pass passive filter 
This filter capacitor uses triangle connection, not only reducing capacitance value but also suppress three times harmonic. It can provide low impedance in specific frequency bands to filter out the harmonic without a filter device in each harmonic resonance point [9]. The circuit shown in Fig. 2 can be simplified into single-phase $Y$ impendence circuit as (2).

$$
Z=-j \frac{9 \omega^{4} L_{1} L_{2} C_{1} C_{2}-3 \omega^{2}\left[L_{2} C_{2}+L_{1}\left(C_{1}+C_{2}\right)\right]+1}{3 \omega^{2}\left(C_{1}+C_{2}-3 \omega^{2} L_{2} C_{1} C_{2}\right)}
$$

In order to provide low channel impendence for different frequency higher harmonics, the numerator of the equation must be zero. Different harmonic frequency can be calculated through equntion3.

$$
\begin{aligned}
& H_{a}=\frac{\omega}{2 \pi \times 50}=\frac{\omega}{2 \pi \times 50 \times 2 \times 9 \times L_{1} L_{2} C_{1} C_{2}} \\
& \times\left\{\begin{array}{l}
3 L_{2} C_{2}+3 L_{1}\left(C_{1}+C_{2}\right) \\
+\left\{\begin{array}{l}
{\left[3 L_{2} C_{2}+3 L_{1}\left(C_{1}+C_{2}\right)\right]^{2}} \\
-\frac{4 \times 9 \times L_{1} L_{2} C_{1} C_{2}}{2 \times 9 \times L_{1} L_{2} C_{1} C_{2}}
\end{array}\right\}
\end{array}\right\}
\end{aligned}
$$

\section{CURRENT CONTROL OF HAPF AND SVC XOMPREHENSIVE COMPENSATION CONTROL SYSTEM}

In order to solve the two key problems of harmonic current pollution and reactive compensation, a combined compensation system of HAPF and SVC is proposed, which is composed of a static var compensator and an active power filter for unbalanced three-phase distribution feeders with harmonic distortion, suited to be installed in a point of common connection[10]. In this system, the SVC acts as a classic reactive power compensator for load balancing and power factor correction, added low capacity resonant impendence type hybrid active filter, RITHAF. RITHAF is in parallel with the resonance branch of fundamental wave through the coupling transformer, then series with the fixed capacitor of SVC structure to connect to the grid. The small rating active power filter is used to improve filtering characteristics of the passive power filter in SVC and suppress possible resonance between the system impedance and the passive power filter. The single phase equal model of combined compensation system is shown in Fig. 3.

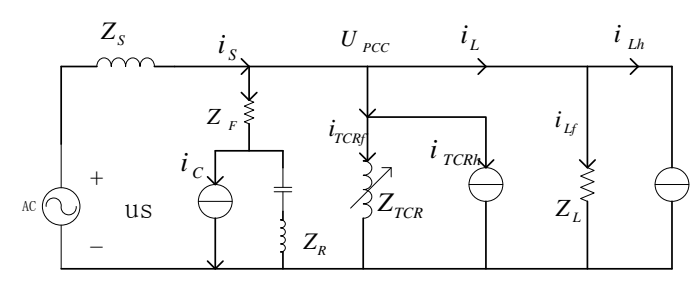

Fig. 3. single phase equal model of combined compensation system impendence equivalences as the voltage source, the following equation can be listed based on the methods of Node Voltage and KCL.

$$
\left\{\begin{array}{l}
\left(\frac{1}{Z_{S}}+\frac{1}{Z_{F}+Z_{R}}+\frac{1}{Z_{T C R}}+\frac{1}{Z_{L}}\right) U_{P C C} \\
=\frac{U_{S}}{Z_{S}}-\frac{I_{C} Z_{R}}{Z_{F}+Z_{R}}-I_{T C R h}-I_{L h} \\
I_{S}=I_{F}+I_{T C R}+I_{L}
\end{array}\right\}
$$

The node voltage can be calculated, then the corresponding branch current according above equations. The current of $i_{T C R}$ and $i_{L}$ can be split for the two parts of fundamental and harmonic current, substituting into the equation to derivate equation 5 .

$$
\begin{aligned}
& I_{S}=\left(\frac{1}{Z_{F}+Z_{R}}+\frac{1}{Z_{T C R}}+\frac{1}{Z_{L}}\right) U_{P C C} \\
& +\frac{I_{C} Z_{R}}{Z_{F}+Z_{R}}+I_{T C R h}+I_{L h}
\end{aligned}
$$

In order to make this system effectively filter harmonic current, the output current is as equation5 by control the active part of active filter simply.

$$
I_{C}=\left(-\frac{Z_{F}}{Z_{R}}-1\right)\left(I_{T C R h}+I_{L h}\right)
$$

\section{COMPARISON OF HARMONIC SUPPRESSION REALIZATION MEANS OF SMART DisTRIBUTION NETWORK WITH GRID-CONNECTED PHOTOVOLTAIC GENERATION}

TABLE I: THE CHARACTERISTICS OF HARMONIC SUPPRESSION REALIZATION

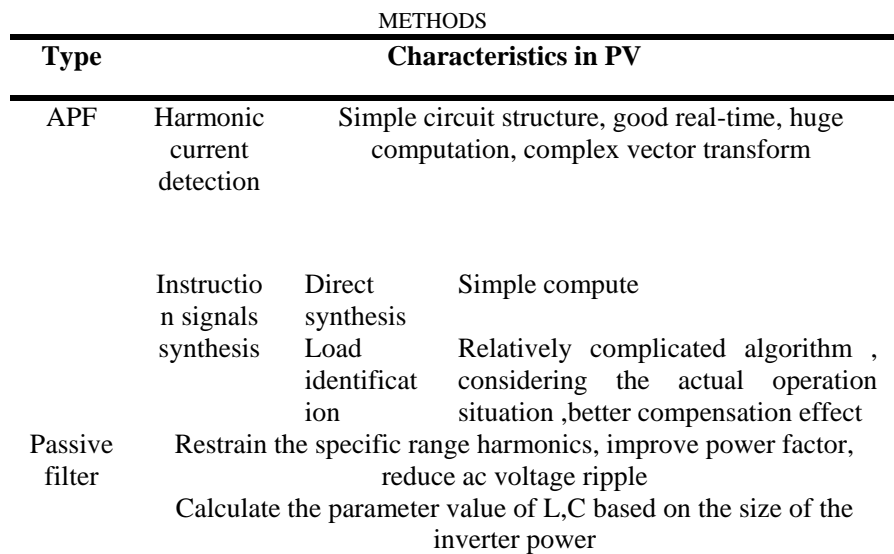
electronic devices, rectifier circuit phase delay

The parallel branch of current source and electrical 
Table I lists the characteristics of above harmonic suppression realization methods of smart distribution network with PV system. In addition, Unified Power Quality Conditioner , UPQC controls the harmonic and voltage volume at the same time, is a hot research area. Many different factors should be considered in practical application of harmonic suppression, such as: load condition, the actual capacity, control plants, system type, economic cost, etc [11]. comparison of harmonic suppression realization means of smart distribution network with grid-connected photovoltaic generation

\section{ORECAST AND SUMMARIES}

Adopt more effective compensation signal extraction algorithm. With the development of computer technology, digital control technology and DSP, it has becoming to be focused that methods based on Fourier, wavelet analysis and neutral network.

Design corresponding synthesis algorithm in accordance with different practical situations. As the capacity of the inverter in PV grid system is limited in practical application, we should choose corresponding algorithm to control the inverter work based on the solar energy power generation, load and power quality pollution situation.

According to the phase delay of output current of combined compensation system which is composed of hybrid active power filter (HAPF) and static var compensator (SVC), Applying generalized integrators with excellent performances of frequency division, a generalized integral control based on predictive compensation at different frequency for generalized delay is proposed. a novel $\pi$-aimed Smith predictor is established to realize the effective compensation for generalized delay in paper.

\section{REFERENCES}

[1] Z.-K. Shuai, A. Luo, D.-G. Liu et al., "Combined system of static var compensator and active power filter," in Proc. the CSEE, 2009, vol. 29, no. 3, pp. 56-64.

[2] P.-C. Qiu, B.-M. Ge, D.-Q. Bi, "Battery energy storage-based power stabilizing control for grid-connected photovoltaic power generation system," Power System Protection and Control, 2011, vol. 39, no. 3, pp. 29-33.

[3] N.-C. Zhou, L.-W. Yan, Q.-G. Wang, "Research on dynamic characteristic and integration of photovoltaic generation in micro grids," Power System Protection and Control, 2010, vol. 38, no. 14, pp. 119-127.

[4] Y. Zhang, Q. Li, Z.-H. Li et al., "Conceptual design for the project of micro grid system interconnected with photovoltaic generation \& energy storage," Power System Protection and Control, 2010, vol. 38, no. 23, pp. 212-214.

[5] Y. Zhao and X.-H. Hu, "Impacts of distributed generation on distribution system voltage sags," Power System Technology, 2008, vol. 32 , no. 14 , pp. 5-18

[6] C.-S. Du, C.-H. Zhang, X.-Z. Liu et al., "Control strategy on the three-phase grid-connected photovoltaic generation system with shunt active power filter," Transactions of China Electro technical Society, 2010, vol. 25, no. 9, pp. 163-169.

[7] T.-F. Wu, H.-S. Nien, H.-M. Hsieh et al., "PV power injection and active power filtering with amplitude-clamping and amplitude-scaling algorithms," IEEE Transactions on Industry Applications, 2007, vol. 43, no. 3, pp. 731-741.

[8] C.-M. Tu, A. Luo, "Iterative generalized integrator algorithm based ternary variable structure control for active power filter," Advanced Technology of Electrical Engineering and Energy, 2004, vol. 23, no. 1, pp. 34-38.

[9] R.-X. Fan, A. Luo, K. Zhou et al., "The modeling and the control strategy analysis of shunt hybrid active power filters," in Proc. the CSEE, 2006, vol. 26, no. 12, pp. 55-61.

[10] X. Yuan, W. Merk, H. Stemmler et al., "Stationary frame generalized integrators for current control of active power filters with zero steady state error for current harmonics of concern under unbalanced and distorted operating conditions," IEEE Trans. on Industry Applications, 2002, vol. 38, no. 2, pp. 523-532.

[11] X. Jin, L.-J. Dong, S.-M. Wu et al., "Implementation of an improved method of harmonic current detection," Power System Protection and Control, 2010, vol. 38, no. 3, pp. 39-42.

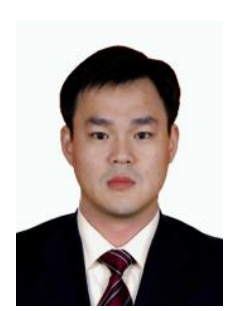

Lifeng Zhu was born in Qufu, Shandong Province, in October, 1975. He has received the Bachelor's degree in electrical engineering from North China Electric Power University in June 1998. He works at Shandong Electric Power Group Corporation, Jining Power Supply Company. His location is Senior Engineer. Previous publications: Rural Substation Voltage Regulation and Reactive Power Compensation Package (Qufu,Shandong Province, Lifeng Zhu,2009). The Application of Distribution Automation in Distribution Production Management (Qufu,Shandong Province, Lifeng Zhu,2009). His current interests are smart gird and power system digital protection. 